women receiving physical examinations for any HPV subtypes, HR HPV subtypes, 9-valent HPV and 4-valent HPV.

Conclusion Our study delineated the distribution and trend of type-specific HPV among both gynecological outpatients and women receiving physical examinations in Guangdong, which may provide valuable data to inform cervical cancer screening and HPV vaccination programs for women in this province.

Disclosure No significant relationships.

\section{P835 QUANTITATIVE ORAL HPV16 AND HPV18 DETECTION IN PATIENTS ATTENDING DENTAL CLINICS}

${ }^{1}$ Helen Stankiewicz Karita*, ${ }^{2}$ Amalia Magaret, ${ }^{3}$ Quinne Feng, ${ }^{4}$ Anna Wald. ${ }^{1}$ University of Washington, Seattle, USA; ${ }^{2}$ University of Washington; Fred Hutchinson Cancer Research Center, Department of Biostatistics and Department of Laboratory Medicine, University of Washington; Department of Public Health Science, Fred Hutchinson Cancer Research Center, Seattle, USA; ${ }^{3}$ Fidalab, Seattle, USA; ${ }^{4}$ University of Washington; Fred Hutchinson Cancer Research Center, Department of Medicine, Department of Laboratory Medicine, Department of Epidemiology, University of Washington; Vaccine and Infectious Diseases Division, Fred Hutchinson Cancer Research Center, Seattle, USA

10.1136/sextrans-2019-sti.880

Background The incidence of HPV-associated oropharyngeal cancer is increasing substantially, especially among men. Our goal was to assess quantitative HPV16 and HPV18 detection in oral rinses obtained in dental offices in Seattle, Washington. Methods We evaluated 15,313 oral rinses collected for during routine dental visits from 11/2016 to 11/2018. Multiplex Taqman qPCR was utilized to determine HPV16 and HPV18 viral load (VL).

Results In persons with a single sample, oral HPV was detected in $152(1 \%)$ persons: $127(0.83 \%)$ were HPV16 positive and $25(0.16 \%)$ were HPV18 positive. HPV16 was detected in $1.4 \%$ of men; the median age was 55 and median VL was 39.7 (range 0.1 - 589855.2 copies $/ \mathrm{mL}$ ). Only $0.4 \%$ of samples were HPV16 positive in women (median age 48, median VL 1.08, range 0.01 - 825 copies $/ \mathrm{mL})$. HPV18 was detected in $13(0.18 \%)$ men and $12(0.14 \%)$ women. A second oral rinse was collected in 628 persons (mean 6 months apart): 581 were HPV negative at baseline and only one became HPV16 positive at second rinse, 39 were HPV16 positive at baseline and 13 remained HPV16 positive at the second rinse, and 8 were HPV18 positive at baseline and 2 remained HPV18 positive at subsequent rinse. Patients with consecutive positive tests were all men and had higher baseline median VL (385 vs 0.90 HPV16 copies/mL; 24 vs 0.80 HPV18 copies $/ \mathrm{mL}$ ) compared to those with first positive and second negative samples.

Conclusion Oral rinse is an acceptable method of HPV testing and patients seen for routine dental care are interested in testing. Comparable to published studies, oral HPV was more frequent among men than women, especially at higher VL levels. HPV16 persistence was more common in those with high VL at baseline test. Future studies are needed to evaluate the feasibility of an effective primary and secondary screening strategy for oropharyngeal cancer using quantitative oral HPV detection.

Disclosure No significant relationships.
P836

TRENDS IN ANOGENITAL WARTS SINCE INTRODUCTION OF HUMAN PAPILLOMAVIRUS VACCINES IN CONNECTICUT, USA

${ }^{1}$ Linda Niccolai*, ${ }^{2}$ Monica Brackney. ${ }^{1}$ Yale School of Public Health, Epidemiology of Microbial Diseases, New Haven, USA; ${ }^{2}$ Yale School of Public Health, CT Emerging Infections Program, New Haven, USA

\subsection{6/sextrans-2019-sti.88}

Background HPV vaccines have been available in the US since 2006 and have the potential to prevent $\geq 90 \%$ of anogenital warts (AGW). Monitoring trends in AGW is important to assess progress of immunization programs.

Methods Two datasets for Connecticut (population 3.6 million) were used. Data for residents with private insurance were available for 2012-2017 from a multi-payer claims database $(\sim 1$ million covered individuals per year). Data for residents with Medicaid insurance were available for 2009-2013 $(\sim 512,000$ individuals per year).

Results Among privately insured women, the annual incidence rate (IR) of AGW declined from 104 to 68 per 100,000 during 2012-2017. Significant declines were observed for women aged 15-19 ( $\mathrm{p}$ for trend $=.03$, average annual percent change (AAPC) -11\%), 20-24 (p<0.001, AAPC -13\%), 25-29 $(\mathrm{p}<0.001$, AAPC $-7 \%)$, and 30-34 $(\mathrm{p}<0.01$, AAPC $-2 \%)$. Similar patterns were observed among men, with an overall decline from 179 to 105 per 100,000 , and $p<0.001$ for trends in each age group and AAPCs ranging from $-16 \%$ to 3\%. Among Medicaid-insured women, the overall IR of AGW declined from 175 to 145 per 100,000 during 2009-2013. Significant declines were observed for women up to age 29 years $(\mathrm{p}<0.05$ and AAPCs from $-13 \%$ to $-3 \%$ ). Rates in Medicaid-covered men did not decline in any age group.

Conclusion In Connecticut, significant and substantial declines in AGW have occurred in women during both periods (20092013 and 2012-2017). In men, declines occurred during 2012-2017 but not during 2009-2013, perhaps due in part to the later routine recommendation for males in $2011 \mathrm{com}$ pared to females in 2006. Greater declines in younger populations are consistent with HPV vaccine impact. These reductions have been achieved in a setting of moderate HPV vaccine uptake and could be further reduced with higher coverage.

Disclosure No significant relationships.

\section{P837 HPV16/18 VACCINE: INFLUENCE ON THE SYSTEMIC AND LOCAL TH1/TH2 CYTOKINE PROFILE}

${ }^{1}$ Paulo Giraldo, ${ }^{2}$ José Sanches, ${ }^{1}$ Rose Luce Do Amaral ${ }^{*},{ }^{1}$ Isabel Migliorini De Oliveira, ${ }^{2}$ Cristiane Gil, ${ }^{1}$ Michelle Discacciati. ${ }^{1}$ UNICAMP, Faculdade De Ciências Médicas, Campinas, Brazil; ${ }^{2}$ UNIFESP, Departamento de Morfologia e Genética, São Paulo, Brazil

\subsection{6/sextrans-2019-sti.882}

Background The immunological mechanism of the vaccines acts systemically in order to prevent a specifically infection. Although HPV-antibodies levels have been studied in serum and cervicovaginal (CVC) samples of vaccinated women, the TH1/TH2 cytokines levels has not yet been adequately characterized. In our study, we investigated the effects of the 